\title{
Displasia fibromuscolare delle arterie renali: aspetti clinici e diagnostica ultrasonografica
}

\author{
Antonio Mannarino \\ Nefrologo Libero Professionista, Humanitas PAS, Scandicci (FI)
}

\begin{abstract}
Renal artery fibromuscular dysplasia: clinical aspects and ultrasound diagnostics
Fibromuscular dysplasia (FMD) is a noninflammatory and nonatherosclerotic type of vascular stenosis that affects mainly the renal and carotid arteries. The most common type of FMD is the media fibroplasia with the characteristic "string of beads" appearance. The disease must be suspected in young people, commonly women, with arterial hypertension, in the resistant hypertension and in the hypertension associated with a decrease of renal mass. Considering that the string of beads appearance generally isn't seen on duplex imaging of renal arteries, a peak systolic velocity of $285 \mathrm{~cm} / \mathrm{sec}$ or a renal aortic ratio of 3.5 alone can be used in detecting $>60 \%$ of renal artery stenosis. Duplex ultrasound visualization of middle third of renal arteries may be very difficult in some cases, particularly on the left side as a result of gas artifact from the colon. In these cases comparing spectral waveforms and resistive index at the renal hilum can be helpful. Catheter based angiography remains the most accurate imaging to diagnose and evaluate FMD. Balloon angioplasty alone of the renal arteries is the treatment of choice of hypertension due to FMD and should be considered in patients with a recent onset of hypertension in whom the goal is the cure of hypertension, in those in whom blood pressure control is difficult to achieve and in those who have lost renal volume because of ischemic nephropathy.
\end{abstract}

Keywords: Fibromuscular dysplasia, Renal artery angioplasty, Renal color duplex ultrasound

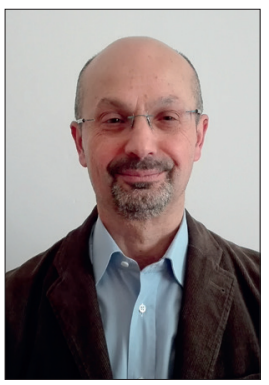

\section{Introduzione}

La Displasia Fibromuscolare (FMD) è una malattia vascolare non infiammatoria e non aterosclerotica che interessa principalmente le arterie renali e le carotidi interne ma, anche se raramente, sono state descritte lesioni fibrodisplasiche in tutti i distretti vascolari (1). La prevalenza della malattia viene considerata bassa nella popolazione generale e le cause e la fisiopatologia della FMD sono ancora poco note. La presentazione clinica assume diversi aspetti, da forme asintomatiche a un quadro vascolare

Accepted: March 2, 2017

Published online: May 2, 2017

Indirizzo per la corrispondenza:

Dr. Antonio Mannarino

Nefrologo Libero Professionista

PAS Humanitas

Via Bessi, 2

50018 Scandicci (FI)

amannarino1@alice.it multi-sistemico simile alle vasculiti, con espressione clinica variabile a seconda del distretto vascolare interessato, del grado di stenosi e del tipo di fibrodisplasia. Dal momento che le arterie renali sono le arterie più frequentemente coinvolte, l'ipertensione arteriosa in età giovanile rappresenta il quadro clinico d'esordio più comune.

La malattia in alcuni casi può assumere però un carattere di gravità per complicanze neurologiche legate alla possibile localizzazione vascolare cervico-encefalica.

\section{Epidemiologia e Clinica}

La FMD nelle arterie renali è stata osservata nel 6.6\% dei soggetti sottoposti ad angiografia allo scopo di donazione di rene, mentre la localizzazione nelle arterie cervico-encefaliche è stata descritta con una frequenza più bassa (0.3-3\%) (2).

Nella FMD le arterie renali sono interessate nel 60-75\% dei casi in modo unilaterale e nel 35\% dei casi in modo bilaterale. Da tenere presente che, di questi pazienti, il 60\% presenta lesioni fibrodisplasiche sulle carotidi o sulle vertebrali e, per questo motivo, in presenza di diagnosi di FMD delle arterie renali, è buona norma effettuare una diagnostica di imaging sul distretto vascolare carotideo ed encefalico per escludere la presenza di lesioni sui vasi extracranici e di aneurismi intracranici. 
Tra le cause di stenosi dell'arteria renale, la FMD è responsabile di poco meno del $10 \%$ di tutti i casi mentre la forma aterosclerotica ne rappresenta la quota nettamente più rilevante (circa il 90\%). Esiste una netta prevalenza del genere femminile (91\%) in una fascia d'età compresa tra i 15 e i 50 anni. Le cause della malattia rimangono poco note sebbene siano state avanzate correlazioni con l'abitudine al tabagismo e la presenza di ipertensione arteriosa. Fattori genetici e sicuramente il genere femminile rappresentano condizioni predisponenti allo sviluppo della malattia. La storia naturale della FMD è stata oggetto di diversi studi angiografici che hanno mostrato come le lesioni fibrodisplasiche possano rimanere nel tempo stabili o progredire, $\mathrm{ma}$ in nessun caso regredire (3). La progressione della malattia definita come la comparsa di nuove lesioni, il peggioramento della stenosi e l'ingrandimento di aneurismi si osserva in una percentuale che arriva fino al $37 \%$ dei pazienti. Tuttavia, contrariamente alla stenosi aterosclerotica, la FMD di per sé generalmente non determina l'ostruzione totale arteriosa e, quindi, un infarto renale, a meno che non si complichi con la dissezione di parete (4). In ogni caso la stenosi e l'ipoperfusione parenchimale possono determinare, in una percentuale non trascurabile dei casi (fino al 63\%), un danno parenchimale cronico ischemico che determina una riduzione volumetrica del rene e una riduzione dello spessore del parenchima renale. Rara comunque è l'insorgenza di una nefropatia cronica con riduzione della funzione renale (5). Veri e propri aneurismi dell'arteria renale (circa 5-10\% dei soggetti con fibrodisplasia) e la dissezione rappresentano complicanze della FMD e non fanno parte del quadro tipico istopatologico.

Complica il corretto inquadramento diagnostico il dato che le lesioni fibrodisplasiche possono coesistere con quelle aterosclerotiche. Si tratta di forme di FMD all'esordio asintomatiche, che, nell'età avanzata, si complicano con il sovrapporsi di lesioni aterosclerotiche. Questi soggetti tipicamente presentano la coesistenza nell'arteria renale di lesioni ostiali/ prossimali aterosclerotiche e di lesioni fibrodisplasiche a carico del tratto medio/distale.

\section{Modalità di classificazione}

La classificazione classica delle varianti di FMD, rivisitata da Stanley, si basa sulla tipologia della tonaca vascolare principalmente coinvolta dalla lesione ed è pertanto una classificazione di tipo patologico: FMD intimale (FMDi), FMD mediale (FMDm) e FMD avventiziale (FMDa) (6).

La FMDm è la più comune (80-90\% dei casi) ed è caratterizzata dalla rarefazione nella tonaca media delle cellule muscolari lisce con la sostituzione di tessuto fibroso.

L'intima, la lamina elastica interna e l'avventizia non presentano alterazioni. Si presenta con il classico aspetto angiografico "a corona di rosario" dovuto all'alternanza di zone multifocali di stenosi e di microaneurismi (cioè di dilatazioni post stenotiche). La lesione, bilaterale nel $60 \%$ dei casi, interessa tipicamente il tratto medio e il tratto distale dell'arteria renale. Si osserva soprattutto in donne con un'età tra i 30 e i 50 anni.

La FMDi è meno comune della precedente (5-10\%) ed è caratterizzata da una proliferazione sub-endoteliale di tessuto connettivale che determina, a livello intimale, un restringimento fibroso con una lamina interna duplicata o frammentata. Si osserva in una fascia d'età più giovane (<30 anni) e si presenta come una stenosi concentrica focale o tubulare.

La FMDa ha una frequenza simile alla forma intimale (5-10\%) con la quale condivide anche l'aspetto angiografico. è caratterizzata da un'eccessiva deposizione di tessuto elastico nell'area esterna della media (da cui il nome anche di perimediale). La classificazione patologica non identifica però entità ciniche distinte, dal momento che i diversi tipi di lesione possono coesistere nello stesso paziente. Un superamento di questo aspetto è la successiva classificazione di tipo morfologico di Kincaid, basata su dati angiografici (7):

a) multifocale (multiple stenosi con aspetto "string of beads");

b) unifocale (breve singola stenosi $<1 \mathrm{~cm}$ );

c) tubulare (singola stenosi $>1 \mathrm{~cm}$ );

d) forme miste.

Nel 2012 Savard propose un'ulteriore semplificazione della classificazione morfologica:

- $\quad$ multifocale (presenza di $\geq 2$ stenosi sulla stessa arteria con o senza l'aspetto di "string of beads");

- $\quad$ unifocale (presenza di una singola stenosi focale o tubulare) (8).

\section{Diagnostica}

L'angiografia meglio di qualunque altro esame è in grado di svelare con accuratezza la presenza di stenosi, di aneurismi e di dissezione lungo l'intera lunghezza dell'arteria renale, dall'ostio fino ai rami di divisione intra-renale. Tuttavia l'esame angiografico per la sua invasività non può rappresentare l'indagine di prima scelta nella diagnosi di FMD.

Normalmente sono utilizzate per la diagnosi tecniche meno invasive di imaging, l'Ultrasonografia Duplex Colordoppler (CDUS), I'Angio-TC (CTA) e l'Angio-RMN (MRA). La CTA e la MRA presentano un'elevata accuratezza diagnostica (sensibilità e specificità intorno al $95 \%$ ) e sono ampiamente utilizzate nella diagnosi di stenosi, aneurismi e dissezione presenti nella FMD. Ambedue vengono generalmente eseguite dopo un esame CDUS positivo o in caso esame CDUS negativo o dubbio, in presenza di un elevato sospetto clinico di malattia. La CTA possiede una migliore risoluzione spaziale rispetto alla MRA; ambedue le tecniche non consentono la visualizzazione ottimale dei rami di divisione dell'arteria renale; la MRA, a causa di una risoluzione spaziale più bassa e dei movimenti 
del paziente, può determinare la visualizzazione dell'aspetto a "corona di rosario" laddove non esiste. In ogni caso le due tecniche per vari motivi (uso di contrasto iodato/gadolinio, costi dell'esame, disponibilità delle apparecchiature, tossicità da contrasto) sono considerati esami di secondo livello nella diagnosi della FMD. La CDUS ha il vantaggio di essere facilmente eseguibile, incruenta, non dannosa, ripetibile e non limitata dalla presenza di insufficienza renale. Pur con dei limiti, in parte legati alla capacità dell'operatore e in parte legati alle caratteristiche del paziente (obesità, meteorismo addominale, collaborazione), la CDUS rappresenta la metodica di imaging di primo livello nella diagnosi della stenosi dell'arteria renale.

La metodica ecografica inoltre riveste un ruolo assolutamente primario nel follow-up dei pazienti sottoposti a rivascolarizzazione endovascolare. Nella CDUS i criteri utilizzati per la diagnosi di stenosi fibromuscolare dell'arteria renale sono quelli classici utilizzati per la diagnosi della stenosi aterosclerotica:

a) diretti, rilevati in sede di stenosi e b) indiretti, rilevati a valle della stenosi.
I criteri diretti sono la Velocità di Picco Sistolico (PSV) in $\mathrm{cm} / \mathrm{sec}$ e il Rapporto PSV arteria renale/PSV aorta (RAR) (9). Tra i criteri indiretti, il Tempo di Accelerazione (AT) in msec (valore normale $<70$ ) e la differenza tra gli Indici di Resistenza $(\Delta \mathrm{RI})$ tra i due reni (valore normale $<0.06$ ) sono quelli che rivestono maggiore importanza (10).

Il parametro AT definisce l'intervallo di tempo tra l'inizio della sistole e il picco sistolico precoce, quando presente, e, se assente, dall'esordio della sistole al primo punto di deflessione del tracciato spettrale. II parametro RI, valutato come AT sui vasi segmentari o interlobari, è espressione delle resistenze periferiche e della quota diastolica del flusso tissutale e viene espresso dal rapporto $S-D / S$, dove $S$ è la velocità di picco sistolica e $D$ è la velocità diastolica.

Esistono vari studi di validazione dei criteri Doppler utilizzati per la diagnosi di stenosi. Valori elevati di cut off offrono, a spese di una minore sensibilità, una maggiore specificità e viceversa. In questi casi la scelta ottimale del valore del cut off deve rappresentare un giusto compromesso tra una buona sensibilità e una buona specificità.

Nella Tabella I vengono riassunti alcuni studi di validazione con relativi valori di cut off di positività. La metodica di riferimento è l'angiografia renale accompagnata eventualmente

TABELLA I - Accuratezza dei criteri CDUS validati da controlli angiografici nella diagnosi di stenosi emodinamica dell'arteria renale

\begin{tabular}{|c|c|c|c|c|c|}
\hline Autore & Numero/(DS angio) & Criterio e cut off & Sensibilità & Specificità & Riferimento \\
\hline \multirow[t]{5}{*}{ Abu Rahma (9) } & $313(60 \%)$ & PSV 180 & $91 \%$ & $41 \%$ & RA;TSPG \\
\hline & & PSV $>285$ & $67 \%$ & $90 \%$ & \\
\hline & & RAR $>3.5$ & $72 \%$ & $81 \%$ & \\
\hline & & PSV $180+$ RAR >3.5 & $72 \%$ & $81 \%$ & \\
\hline & & PSV $285+$ RAR >3.5 & $60 \%$ & $94 \%$ & \\
\hline \multirow[t]{2}{*}{ Miralles (11) } & 78 (>60\%) & PSV $>198$ & $87 \%$ & $92 \%$ & RA \\
\hline & & RAR $>3.3$ & $76 \%$ & $92 \%$ & \\
\hline Kohler (12) & $114(60 \%)$ & RAR 3.5 & $91 \%$ & $95 \%$ & RA \\
\hline Kawarada (13) & $94(60 \%)$ & PSV > 219 & $89 \%$ & $89 \%$ & RA;TSPG \\
\hline \multirow[t]{3}{*}{ Conkbayr (14) } & 50 (>60\%) & PSV $>180$ & $89 \%$ & $88 \%$ & RA \\
\hline & & RAR $>3$ & $86 \%$ & $97 \%$ & \\
\hline & & $\mathrm{TA}>70 \mathrm{msec}$ & $48 \%$ & $93 \%$ & \\
\hline \multirow[t]{3}{*}{ Straub (16) } & 49 (>70\%) & PSV $>250$ & $89 \%$ & $70 \%$ & RA;TSPG \\
\hline & & RAR $>3.5$ & $84 \%$ & $72 \%$ & \\
\hline & & $\Delta \mathrm{RI}>0.05$ & $42 \%$ & $91 \%$ & \\
\hline \multirow[t]{2}{*}{ Drieghe (15) } & 47 (>65\%) & PSV >318 & $88 \%$ & $77 \%$ & $Q A ; I P G$ \\
\hline & & RAR $>3.74$ & $75 \%$ & $97 \%$ & \\
\hline
\end{tabular}

DS angio = diametro della stenosi in angiografia; IPG = gradiente pressione invasivo; PSV = velocità di picco sistolico; $Q A=$ angiografia quantitativa; $R A=$ arteriografia renale; RAR = rapporto PSV renale aorta; TA = tempo di accellerazione; TSPG = gradiente pressione trans-stenotico. 
dal gradiente pressorio trans-stenotico (TSPG) che, se $>20$ $\mathrm{mmHg}$, depone per la presenza di una stenosi emodinamica. Altri Autori hanno validato i criteri Doppler con la misurazione del gradiente invasivo di pressione (IPG) (15). Questa metodica si avvale dell'uso di micro-cateteri (0.0014" pressure wire) che consentono la misurazione diretta della pressione a valle della stenosi $(\mathrm{Pd})$, mentre la pressione in aorta $(\mathrm{Pa})$ viene misurata come di norma con il catetere 4 Fr. Secondo questi Autori, il rapporto $\mathrm{Pd} / \mathrm{Pa}$ è $<0.9$ e quindi una caduta di pressione transstenotica $>10 \%$ depone per la presenza di una stenosi critica cioè con un diametro maggiore del $61 \%$, che corrisponde a una riduzione dell'area della sezione del vaso (cross-sectional area) dell'84\%.

L'esame ecografico nei pazienti con FMD presenta maggiori difficoltà perché viene richiesta la visualizzazione ottimale del tratto medio dell'arteria renale e questa operazione, soprattutto a sinistra, è, a volte, estremamente difficile, se non impossibile, per la presenza di artefatti da meteorismo colico.

Con l'ultrasonografia, anche nelle situazioni di migliore visualizzazione delle arterie renali, non è possibile effettuare una misurazione del diametro del lume residuo del vaso stenotico. Con il Doppler però possiamo calcolare la velocità di flusso all'interno del vaso e quindi risalire all'area di sezione del vaso e al diametro della stenosi (effetto Venturi):

Flusso $(F)=$ Velocità del sangue $(V) \times$ Area di sezione del vaso (A);

(se si riduce $A$, perché $F$ rimanga costante, deve aumentare V).

L'esame CDUS viene completato con la valutazione dei criteri indiretti a valle della stenosi (AT e $\Delta \mathrm{RI}$ ). A livello intrarenale si possono identificare tre tipi di velocitogramma:

- $\quad$ tipo 1, normale, bifasico con rapida ascesa sistolica e presenza del picco sistolico precoce;

- tipo 2a, normale, bifasico con picco sistolico tardivo e ascesa sistolica <70 msec;

- tipo 2b, patologico, bifasico con picco sistolico tardivo e ritardo sistolico (AT >70 $\mathrm{msec}$ );

- tipo 3, patologico, monofasico, in cui è evidente, oltre che il ritardo sistolico (tardus), anche la riduzione della PSV (parvus) (Fig. 1).

Occorre sottolineare che il tracciato spettrale Doppler post stenotico tardus parvus (ridotta PSV e prolungamento AT) può non essere così evidente nei soggetti anziani con aterosclerosi e danno parenchimale renale. II $\Delta \mathrm{RI}$ può essere inoltre espressione di un differente grado di danno parenchimale tra i due reni. Pur con queste limitazioni, AT è lo specchio di una stenosi arteriosa marcata e il valore di cut off di 70 msec possiede una buona specificità (intorno al 93\%) con una più bassa sensibilità (intorno al $50 \%$ ).

Allo stesso modo il $\Delta R I>0.05$ possiede una buona specificità e una più bassa sensibilità $\mathrm{ma}$, nei soggetti con $<50$ anni,
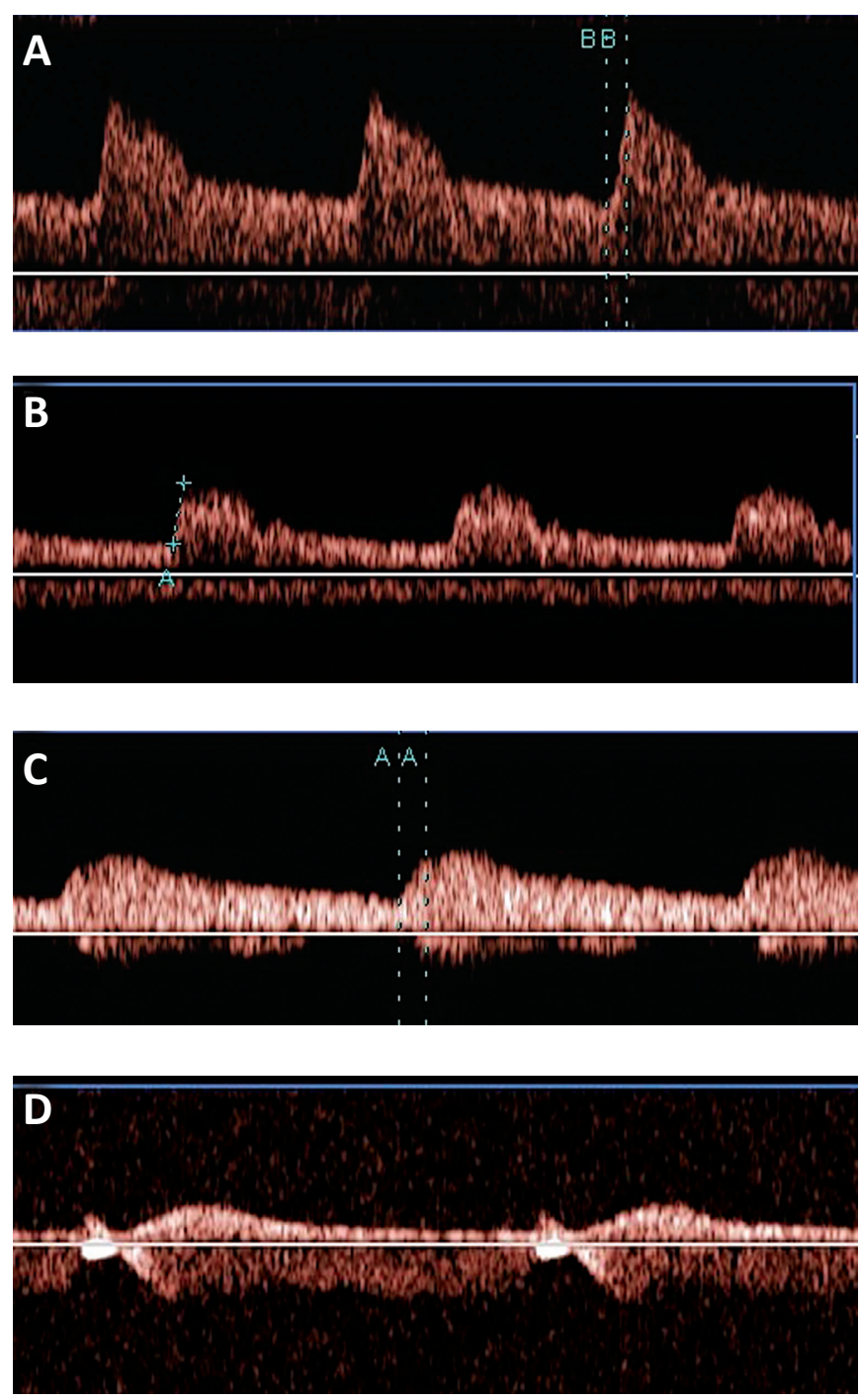

Fig. 1 - Tipologia analisi spettrale intrarenale. (A) Morfotipo 1, bifasico con picco sistolico precoce, normale; (B) Morfotipo 2a, bifasico con picco sistolico tardivo e AT $<70 \mathrm{msec}$, normale; (C) Morfotipo $2 \mathrm{~b}$, bifasico con picco sistolico tardivo e AT >70 msec, patologico; (D) Morfotipo 3, monofasico, patologico.

lo stesso valore di cut off presenta una migliore accuratezza diagnostica (sensibilità $90 \%$ e specificità $99 \%$ per stenosi >75\%) (17). Oltre ai criteri Doppler classici già individuati per la stenosi aterosclerotica, può essere utile, nella forme fibrodisplasiche, il rapporto PSV tra il tratto di arteria stenotico e quello prossimale non stenotico: un rapporto PSV $>2$ indica la presenza di una stenosi $>50 \%$ e un rapporto $>4$ indica una stenosi $>75 \%$ (18).

A scopo esemplificativo a quanto detto, riportiamo il caso di una giovane donna (44 anni) con ipertensione arteriosa, insorta da un anno, giunta alla nostra osservazione. I dati salienti rilevabili all'esame CDUS sono: asimmetria renale ( $\mathrm{dx}$ cm 11 vs sn cm 9.5), spessore del parenchima mediamente 


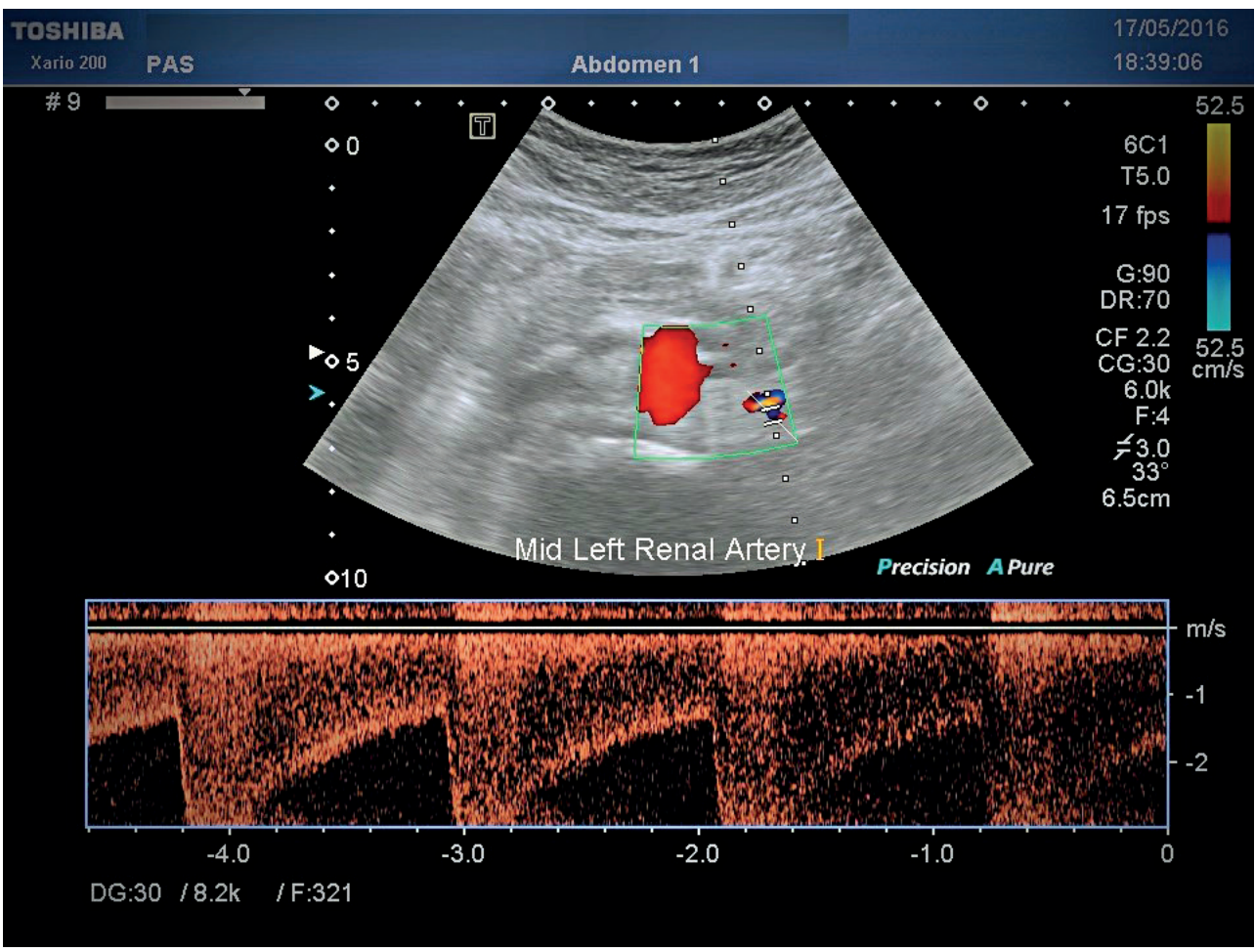

Fig. 2 - Tratto medio arteria renale sn: focalità di aliasing e PSV $400 \mathrm{~cm} / \mathrm{sec}$.

conservato bilateralmente, meglio rappresentato a $\mathrm{dx}$ (17 vs $15 \mathrm{~mm}$ ), arterie renali in $B$ mode all'ostio di calibro normale (5 mm), PSV ostio e tratto prossimale entrambe arterie renali $100 \mathrm{~cm} / \mathrm{sec}$, PSV tratto medio arteria renale $\mathrm{dx} 150 \mathrm{~cm} / \mathrm{sec}$, PSV tratto medio arteria renale sn fino a $400 \mathrm{~cm} / \mathrm{sec}$ (Fig. 2) con RAR $=5.0$ e PSV ratio tratto medio/prossimale $=4.0$, segni indiretti di stenosi $\Delta \mathrm{RI}=0.08$ e AT rene sn $89 \mathrm{msec}$. In questo caso i dati depongono per la presenza di una stenosi emodinamica (intorno al 75\%) del tratto medio dell'arteria renale sn. Una successiva TCA ha evidenziato il classico aspetto "string of beads" del tratto medio e distale dell'arteria renale sn. La paziente successivamente è stata sottoposta con buon esito ad angioplastica senza complicanze peri-procedurali. La coesistenza di una malattia vascolare è stata esclusa nel distretto carotideo-vertebrale e cerebrale (CDUS e RMA). A distanza di 6 mesi dall'angioplastica perdura la sospensione della terapia anti-ipertensiva con sufficiente controllo dei valori di pressione arteriosa.

\section{Trattamento}

Il trattamento delle lesioni fibrodisplasiche dipende da una serie di fattori e può essere conservativo o interventistico, endovascolare o chirurgico. Per i pazienti in cui l'ipertensione è presente da più di 8 anni e la diagnosi di FMD viene fatta in età non giovane, il trattamento di scelta rimane quello conservativo farmacologico dell'ipertensione (19). La procedura di angioplastica, solitamente senza stenting, ha soppiantato la tecnica chirurgica per una serie di ragioni: meno invasiva, meno costosa e associata a minore morbidità. Le principali indicazioni in cui i pazienti vengono selezionati alla rivascolarizzazione sono l'ipertensione arteriosa di recente insorgenza, l'ipertensione arteriosa resistente alla terapia medica e la perdita di parenchima o di funzione renale a causa della nefropatia ischemica. Lo stenting dell'arteria renale viene riservato nei casi in cui il normale gradiente di pressione trans-stenotico, misurato in corso di procedura, non viene ripristinato dall'angioplastica isolata e nei casi in cui interviene come complicanza (pre-esistente o durante la manovra) la dissezione di parete. La tecnica chirurgica viene riservata per il trattamento degli aneurismi, quando la procedura endovascolare non è indicata o non è risolutiva. Una meta-analisi con inclusione di 2630 pazienti ha analizzato l'esito del controllo della pressione arteriosa nei pazienti con FMD sottoposti a rivascolarizzazione renale sia endovascolare che chirurgica (20). I risultati, pur con un'ampia variabilità, mostrano che la guarigione dall'ipertensione è stata ottenuta in circa la metà dei casi e un miglioramento del controllo pressorio o una riduzione del numero dei farmaci sono stati osservati in circa il $40 \%$ dei casi, mentre, nel restante $10 \%$, non si è avuto alcun effetto. Possibili complicanze peri-procedurali (perforazione arteria, dissezione o infarto renale segmentale) possono osservarsi in una percentuale non trascurabile di pazienti (circa il 15\%) (21). Tutti i pazienti con FMD e quindi anche quelli sottoposti ad angioplastica vengono trattati con una terapia antiaggregante con piccole dosi di aspirina. Tuttavia la reste- 
nosi può verificarsi con una frequenza intorno al $25 \%$ dei casi dopo 2 anni dalla procedura e viene trattata con un nuovo trattamento di angioplastica con o senza stenting (22).

\section{Conclusioni}

Nonostante la malattia abbia ricevuto molto interesse negli ultimi anni, la FMD rimane ancora poco conosciuta e ampiamente sottostimata (23). Una diagnostica di imaging, in primo luogo la CDUS, deve essere intrapresa nei seguenti casi:

- ipertensione arteriosa in soggetti giovani, soprattutto se con età <35 anni

- ipertensione arteriosa resistente alla terapia farmacologica ottimale (tre farmaci a dosaggio pieno tra cui un diuretico)

- $\quad$ presenza di soffi addominali e ipertensione

- $\quad$ presenza di lesioni fibrodisplasiche in altri distretti vascolari

- $\quad$ ipertensione arteriosa con presenza di asimmetria renale

La maggiore difficoltà nell'esecuzione dell'indagine CDUS risiede nel fatto che la stenosi comunemente interessa il tratto medio distale delle arterie renali, regione poco accessibile per gli ultrasuoni. Quando è possibile evidenziare la regione stenotica, i valori di cut off che presentano un buon compromesso tra sensibilità e specificità sono $285 \mathrm{~cm} / \mathrm{sec}$ per la PSV e 3.5 per il RAR. Nei casi in cui l'esplorazione del tratto medio dell'arteria è incompleta o nulla, la valutazione si basa sulle alterazioni dell'onda spettrale all'ilo renale, nello specifico con la misura dei parametri AT e $\Delta$ RI.

\section{Disclosures}

Financial support: No financial support was received for this submission.

Conflict of interest: The author has no conflict of interest.

\section{Bibliografia}

1. Wesserly Z, Guerry RL, Klavins JV. Disseminated fibromuscular hyperplasia of vascular channels. Arch Pathol. 1973;96: 179-182.

2. Neymark E, LaBerge JM, Hirose R, et al. Arteriographic detection of renovascular disease in potential renal donors: incidence and effect on donor surgery. Radiology. 2000;214:755-760.

3. Schreiber MJ, Pohl MA, Novick AC. The natural history of atherosclerotic and fibrous renal artery disease. Urol Clin North Am. 1984;11:383-392.

4. Gragg AH, Smith TP, Thompson BH, et al. Incidental fibromuscular dysplasia in potential renal donors: long term clinical follow up. Radiology. 1981:172:145-147.

5. Slovut DP, Olin JW. Fibromuscular Dysplasia. N Engl J Med. 2004;350(18):1862-1871.
6. Stanley J. Renal artery fibrodysplasia. In: Renal Vascular Disease. Novick A, Scoble J, Hamilton G (eds). WB Saunders, London. 1996;21-23.

7. Kincaid OW, Davis GD, Hallerman FJ, et al. Fibromuscular dysplasia of the renal arteries: arteriographic features, classification, and observation on natural history of the disease. Am J Roentgenol Radium Ther Nucl Med. 1968;104:271-282.

8. Savard S, Steichen O, Azarinev A, et al. Association between 2 angiographic subtypes of renal artery fibromuscular dysplasia and clinical charcteristics. Circulation. 2012;126(25):30623069.

9. AbuRahma AF, Srivastava M, Mousa AY, et al. Critical analysis of renal duplex ultrasound parameters in detecting significant renal artery stenosis. J Vasc Surg. 2012;56(4): 1052-1058.

10. Stavros AT, Parker SH, Yakes WF, et al. Segmental stenosis of the renal artery pattern recognition of tardus parvus abnormalities with duplex sonography. Radiology. 1992;184: 487-492.

11. Miralles M, Cairols, Cotillas J, et al. Value of Doppler parameters in the diagnosis of renal artery stenosis. J Vasc Surg. 1996;23: 428-435.

12. Kohler TR, Zierler RE, Martin RL, et al. Noninvasive diagnosis of renal artery stenosis by ultrasonic duplex scanning. J Vasc Surg. 1986;4(5):450-456.

13. Kawarada O, Yokoi Y, Yakemoto K, et al. The performance of renal ultrasonography for the detection of hemodynamically significant renal artery stenosis. Catheter Cardiovasc Interv. 2006;68:311-318.

14. Conkbayir I, Yucesoy C, Edguer T. Doppler sonography in renal artery stenosis. An evaluation of intrarenal and extrarenal imaging parameters. Clin Imaging. 2003;27:256-260.

15. Drieghe B, Madaric J, Sarno G, et al. Assessment of renal artery stenosis: side by side comparison of angiography and duplex ultrasound with pressure measurements. Eur Med J. 2008;29:517-524.

16. Staub D, Canevascini R, Huegli RW, et al. Best duplex sonographic criteria for the assessment of renal artery stenosiscorrelation with intra-arterial pressure gradient. Ultra-schall Med. 2007;28:45-51.

17. Ripolles T, Aliaga R, Morote V. Utility of intrarenal Doppler ultrasound in the diagnosis of renal artery stenosis. Eur J Radiol. 2001;40:54-63.

18. Schaberle W, Leyener L, Schierling W, et al. Ultrasound diagnostics of renal artery stenosis. Gefasschirurgie. 2016;(Suppl.1) 21:S4-S13.

19. Davies MG, Saad WE, Peden EK, et al. The long term outcomes of percutaneous therapy for renal artery fibromuscular dysplasia. J Vasc Surg. 2008;48:865-871.

20. Trinquart I, Mounier-Vehier C, Sapoval M, et al. Efficacy of revascularization for renal artery stenisis caused by fibromuscular dysplasia: a systematic review and meta-analysis. Hypertension. 2010;56:525-532

21. Bonelli FS, McKusick MA, Textor SC, et al. Renal artery angioplasty: technical results and clinical outcome in 320 patients. Mayo Clin Proc. 1995;70:1041-1052.

22. Klow NE, Paulsen $D$, Vatne $K$, et al. Renal artery angioplasty using the coaxial technique: ten years of experience from 591 procedures in 419 patients. Acta Radiol. 1998;39:594-603.

23. Lau JF, Lookstein RA, Olin JW. Renal Fibromuscular Dysplasia Update. Endovascular today. 2012;2:74-79. 